\section{Baumwolle schlägt Synthetik}

\section{Matratzen sind ein wichtiges Reservoir für Allergene der Hausstaub- milbe. Die Allergenlast hängt dabei von Eigenschaften wie dem Feuchtigkeitshaltevermögen und dem Temperaturausgleich ab. In einer Studie wurden diese Zusammenhänge genauer unter die Lupe genommen.}

n den Haushalten von 175 Personen mit Asthmasymptomatik wurden mit Hilfe von speziell ausgerüsteten Staubsaugern Staubproben der Matratzen entnommen und während der Staubentnahme die Luftfeuchtigkeit im Schlafzimmer gemessen. Zusätzlich sollten die Studienteilnehmer per Fragebogen Angaben über Matratzeneigenschaften, Wohnraumbeschaffenheit und Reinigungsgewohnheiten machten. Insgesamt wurden 168 Fragebogen ausgefüllt. Keiner der Teilnehmer benutzte milbendichte Überzüge für Matratze, Kissen oder Decke.

Bei der Auswertung ergab sich, dass ein synthetischer Matratzenoberbezug mit höheren Konzentrationen des Milbenhauptallergens Der $\mathrm{p} 1$ assoziiert war verglichen mit Matratzenoberbezügen aus Baumwolle (2,6 versus 0,8 $\mathrm{\mu g} / \mathrm{g}$ Der p 1). Ein höherer Allergengehalt der Matratze von über $2 \mu \mathrm{g} / \mathrm{g}$ oder gar

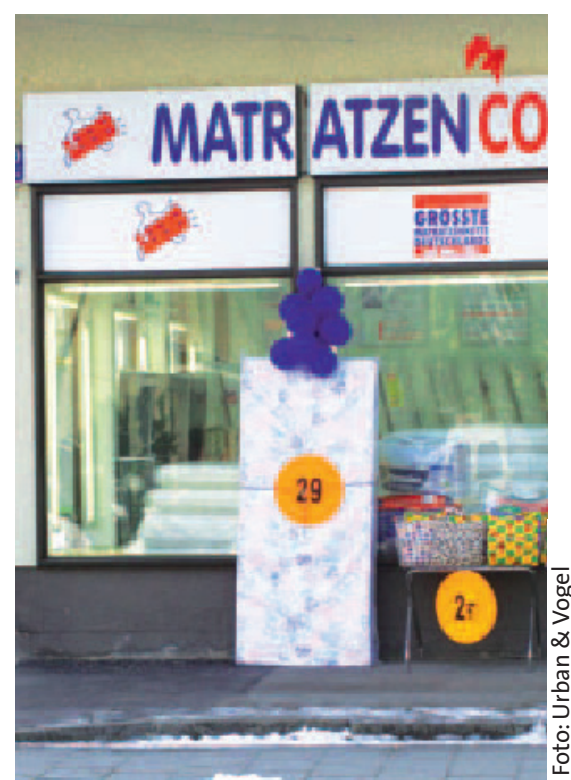

Hausstaubmilbenallergiekranke sind gut beraten, Matratzen mit Synthetiküberzug zu kaufen.
$10 \mu \mathrm{g} / \mathrm{g}$ Staub - dem Schwellenwert zur möglichen Auslösung von Asthmaexazerbationen - war allerdings nicht signifikant mit unterschiedlichen Stoffmaterialien der Oberbezüge assoziiert. Das Material der Matratze selbst - Sprungfeder, Naturlatex, Synthetik oder Wasser - hatte keinen Einfluss auf die Milbenallergenkonzentration, ebensowenig das Matratzenalter, die Temperatur, das Vorhandensein eines Teppichs im Schlafzimmer oder die Benutzung eines Schaffells. Eine signifikante Korrelation ergab sich dagegen zwischen der Höhe der Luftfeuchtigkeit während der Probenentnahme und der Konzentration von Der $\mathrm{p} 1$.

Fazit: Ein Matratzenoberbezug aus Synthetik und eine hohe relative Lufffeuchtigkeit im Schlafzimmer sind mit höheren Konzentrationen von Der p 1 in Matratzen korreliert. Eine ähnliche Assoziation wurde auch schon für Deckenmaterialien gefunden - auch hier waren die Milbenallergenkonzentrationen bei Synthetikmaterialien höher als bei Baumwolle.

Bemt L et al. Influence of mattress characteristics on house dust mite allergen concentration. Clin Exp Allergy 2006; 36: 233-7

beobachtet. In den meisten Fällen berichteten die Patienten über Juckreiz im Mundbereich. Lediglich bei einem Kind traten Rhinorrhoe und Husten auf, bei einem anderen Kind kam es zu Bauchschmerzen und Diarrhoe. Über die klinische Wirkung wird nichts mitgeteilt.

Fazit: Die Ultra-Rush-Dosissteigerung mit sublingual applizierten Inhalationsallergenen war in dieser Studie sicher und gut verträglich. Die Nebenwirkungen beschränkten sich überwiegend auf Juckreiz im Bereich der Mundschleimhaut. Schwerwiegende Nebenwirkungen traten nicht auf.

Tripodi S et al. Safety and tolerability of ultra-rush induction, less than one hour, of sublingual immunotherapy in children. Int Arch Allergy Immunol 2006; 139: 149-52 